\title{
Management of Candida guilliermondii joint infection in a dog
}

\author{
Antonello Bufalari ${ }^{1}$, Chiara Maggio ${ }^{2}$, Giulia Moretti ${ }^{*}$, Alberto Crovace ${ }^{1}$, Valentina Stefanetti ${ }^{1}$, \\ Reinhard Konrad Straubinger ${ }^{3}$ and Fabrizio Passamonti ${ }^{1}$
}

\begin{abstract}
Background: Candida spp. are dimorphic fungi in the family Cryptococcaceae. Infections with Candida spp. are usually rare conditions in dogs, but immunocompromised patients have a higher risk for developing invasive candidal infections.

Case presentation: A 5-year-old male Boxer, positive to Leishmania infantum, was referred to the Veterinary Teaching Hospital of the Department of Veterinary Medicine, University of Perugia, Italy for examination of a non-weight bearing left hind limb lameness of a duration of at least 3 months. During this period, treatment involved systemic anti-inflammatory medications and intra-articular corticosteroid administration. On presentation, clinical examination and radiographic findings were suggestive of cranial cruciate ligament deficiency. To support this diagnosis a stifle arthroscopy was performed: it confirmed a partial rupture of cranial cruciate ligament. Samples culture of synovial fluid and membrane was routinely collected as well, and revealed Candida guilliermondii joint infection. Treatment for the C. guilliermondii joint infection involved systemic anti-fungal therapy, joint lavage and intra-articular administration of antifungal drugs. Lameness improved markedly during this treatment, but lameness did not resolve completely, probably due to cranial cruciate ligament deficiency. Tibial tuberosity advancement (TTA) was chosen in order to treat stifle instability and was performed 4 weeks following cessation of treatment of the C. guilliermondii joint infection. Six month after TTA the dog showed a completely recovery with no lameness.

Conclusions: To the authors' knowledge, this is the first case of Candida spp. joint infection reported in dogs. The cause of the progression of the joint C. guilliermondii infection remains unclear but it may be associated with leishmaniasis or intra-articular corticosteroid injections. Treatment with systemic and intra-articular anti-fungal therapies was successful. In the evaluation of hind limb lameness in a chronically immunocompromised dog, it would be advisable to consider also an intra-articular Candida spp. infection.
\end{abstract}

Keywords: Dog, Joint infectious, Fungal synovitis, Fungal arthritis, Candida guilliermondii

\section{Background}

Candida species are the most frequently cultured fungi in healthy dogs. They are ubiquitous organisms existing almost exclusively as commensal organisms, which rarely become pathogenic [1]. Candida spp. can be isolated in animals from skin and oral, gastrointestinal, upper respiratory, and genital mucosae. In humans, contamination resulting in infection has been reported following

\footnotetext{
${ }^{*}$ Correspondence: giuliamoretti89@gmail.com

1 Department of Veterinary Medicine, University of Perugia, Via S.

Costanzo n.4, 06126 Perugia, PG, Italy

Full list of author information is available at the end of the article
}

iatrogenic compromise of dermal integrity [1]. In human medicine Candida spp. synovitis/arthritis has been associated with intra-articular administration of steroids in order to treat different kind of pathologies such as osteoarthritis and rheumatoid arthritis [2]. The most used intra-articular treatments are methylprednisolone acetate $(40-80 \mathrm{mg})$, triamcinolone hexacetonide (20$40 \mathrm{mg}$ ) and triamcinolone acetonide $(40 \mathrm{mg})$; the duration of treatments depends on the type of disease [2]. Intra-articular glucocorticoids may impair local immune defences. The importance of aseptic technique during intra-articular administration of glucocorticoids, in 
order to decrease the occurrence of secondary infection, has been documented $[2,3]$. Whilst reports of bacterial synovitis/arthritis in dogs are non-uncommon, reports on Candida-associated arthritis have apparently not been published yet, although fungal joint infections have been reported in horses [4-8]. The purpose of this case report is to document a successful treatment with a combination of joint lavage, systemic and local antifungal drugs of a solitary case of a Candida guilliermondii joint infection in a dog.

\section{Case presentation}

A 5-year-old Boxer with a body weight of $30 \mathrm{~kg}$ was admitted to the Veterinary Teaching Hospital of Perugia University, Italy for evaluation of a left hind limb lameness due to a suspected left cranial cruciate deficiency. Lameness had been present for at least 3 months. During that period, treatment was carried out by other veterinarians and had involved systemic anti-inflammatory medication for 2 weeks and intra-articular glucocorticoid administration (drugs and dosages not assessable). Clinical examination revealed reluctance to walk, grade $5 / 5$ lameness based on the Quinn scale [9] and moderate left hind limb muscle atrophy presumed to be secondary to disuse. At rest, the dog demonstrated toe-touching or non-weight bearing left hind limb lameness. Examination revealed a moderate thickening over the medial aspect of the proximal tibia consistent with medial buttress [10]. Manipulations of the joint elicited severe resentment. The cranial drawer and tibial thrust tests were inconclusive. Neurological examination was unremarkable. Examination of the lumbar and lumbosacral spine and hips did not elicit any reaction. After sedation with dexmetedomi$\operatorname{dine}^{1}(4 \mu \mathrm{g} / \mathrm{kg}$ intravenously [IV]), a radiographs of the pelvis and the left hind limb were taken and revealed peri-articular new bone formation in the medial part of the stifle consistent with moderate osteoarthritis and increased synovial fluid volume (effusion). Haematology revealed moderate anaemia (red blood count $[\mathrm{RBC}] 4.87 \times 10^{6}$ cells $/ \mu \mathrm{l}$, Hematocrit $[\mathrm{Hct}] 33.8 \%$, complete blood count $\left.[\mathrm{cBC}] 12.4 \times 10^{3} \mathrm{cells} / \mu \mathrm{l}\right)$ and high level of total proteins $(9.3 \mathrm{~g} / \mathrm{dl})$, while biochemical parameters were within normal range. A possible explanation for the elevated total proteins and anaemia was leishmaniasis and testing subsequently confirmed Leishmania infantum specific antibodies (1/160 Immunofluorescence antibody test [IFAT]). Left stifle arthroscopy was performed. Samples of synovial fluid and synovial membrane and villi were collected and submitted for culture and sensitivity testing. Before premedication with

${ }^{1}$ Dexdomitor $^{\circledR}$ : Orion Corporation Orion Pharma, Espoo, Finland. acepromazine $^{2}(10 \mu \mathrm{g} / \mathrm{kg} \mathrm{IV})$ and methadone ${ }^{3}(0.2 \mathrm{mg} / \mathrm{kg}$ IV), carprofen $^{4}(4 \mathrm{mg} / \mathrm{kg}$, IV) was administered preemptively [11]. General anaesthesia was induced with propofol $^{5}$ (3 $\mathrm{mg} / \mathrm{kg}$ IV), and maintained after endotracheal intubation with a mixture of isoflurane ${ }^{6}$ and oxygen (50$100 \mathrm{ml} / \mathrm{kg} / \mathrm{min}$ ) via a circle breathing circuit with spontaneous ventilation. The left hind limb was prepared for aseptic surgery: trichotomy and aseptic disinfection were performed with a wide margin around the left stifle extending from the proximal third of the femur to the distal third of the tibia. Prior to arthroscopy samples of synovial fluid were collected by needle arthrocentesis after a small $(8 \mathrm{~mm})$ stab incision. The arthroscopic examination was performed using an arthroscope ${ }^{7}$ connected to a video camera and image-recording device. ${ }^{8}$ The arthroscope was introduced at the level of Gerdy's tubercle, $2 \mathrm{~mm}$ lateral to the patellar tendon. The joint cavity was carefully explored and three samples of synovial membrane were gathered with a $2 \mathrm{~mm}$ biopsy forceps. Menisci were examined by visualization and probing as recommended [12]. A small longitudinal tear was identified in the cranial horn of the medial meniscus, more precisely in the cranial tibial ligament of the medial meniscus nearby the intermeniscal transverse ligament. In addition, the craniomedial band of cranial cruciate ligament was ruptured, while the caudolateral band appeared grossly intact. The joint was flushed continuously during joint exploration with sterile saline using a proximal medial parapatellar outflow cannula. At the end of surgery, the arthroscope and surgical instruments were removed and the joint was flushed copiously with lactated Ringer's solution [13]. After recovery from anaesthesia, to provide postoperative analgesia, buprenorphine $^{9}(0.015 \mathrm{mg} / \mathrm{kg}$ subcutaneously [SC]) was administered three times a day [TID] for 1 day while carprofen (see footnote 4) $(2 \mathrm{mg} / \mathrm{kg} \mathrm{SC})$ was given once a day for 10 days. Laboratory findings of the synovial fluid are reported in Table 1.

Smears from synovial fluid were stained with May Gruenwald-Giemsa and examined under a light microscope. The sample showed a blue proteinaceous background and a mixed inflammatory cell population, with some

\footnotetext{
${ }^{2}$ Prequillan $^{\circledR}$, Fatro Spa, Bologna, Italy.

${ }^{3}$ Eptadone $^{\circledR}$, Molteni Spa, Firenze, Italy.

${ }^{4}$ Rimadyl ${ }^{\circledR}$, Zoetis Inc. New Jersey, Stati Uniti.

${ }^{5}$ Proposure $^{\circledR}$, Merial Italia Spa, Milano, Italy.

${ }^{6}$ Isoflo; Esteve Spa, Milano, Italy.

$72.7 \mathrm{~mm}, 30^{\circ}$ oblique arthroscope, Small Joint Arthroscope: Karl Storz GmbH \& Co. KG, Tuttlingen, Germany.

${ }^{8}$ Digital Video Camera DC-1300, NeoMed ${ }^{\circledR}$, Gutach im Breisgau, Germania; Keymat DVD + RW video recorder, Nola, Italy.

9 Temgesic $^{\circledR}$, RB Pharmaceuticals Limited, Milano, Italy.
} 
Table 1 Laboratory findings of the synovial fluid

\begin{tabular}{lllllll}
\hline Colour & Appearance & Quantity $(\mathbf{m l})$ & Protein $(\mathbf{g} / \mathbf{d l})$ & WBC $($ cells $/ \boldsymbol{\mu l})$ & RBC $($ cells/ $\boldsymbol{\mu l})$ & Glucose $(\mathbf{m g} / \mathbf{d l})$ \\
\hline Reddish & Cloudy & 12 & 3.5 & 35,000 & 250 & $<10$ \\
\hline
\end{tabular}

$W B C$ white blood count, $R B C$ red blood count

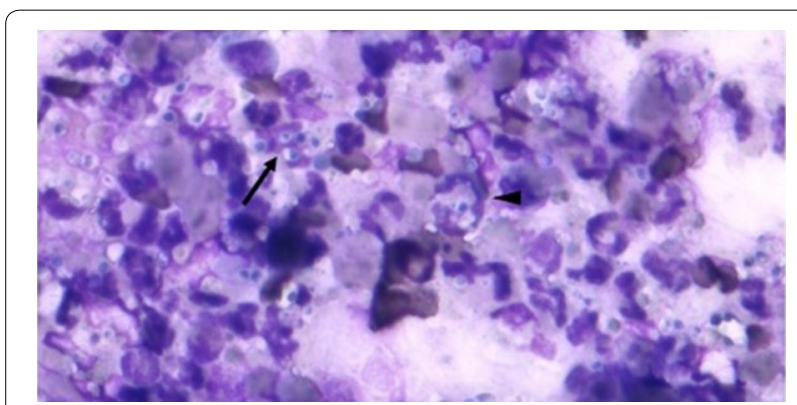

Fig. 1 Cytological appearance of the synovial fluid. 4-6 $\mu \mathrm{m}$ wide yeasts (arrow) and some neutrophils containing yeasts (arrowheads) were observed with May-Grünwald-Giemsa

yeasts as shown in Fig. 1. Synovial fluid and small fragments of the capsule were pre-enriched on $5 \mathrm{ml}$ of triptic soy broth (TSB) medium and incubated at $37^{\circ} \mathrm{C}$. After $48 \mathrm{~h}, 100 \mu \mathrm{l}$ of each broth cultures was subcultured for aerobic bacteria and fungi on blood agar, MacConkey agar and Sabouraud dextrose agar at $37{ }^{\circ} \mathrm{C}$ in $5-10 \%$ $\mathrm{CO}_{2}$. No bacterial growth was observed but a massive growth of yeast with the morphology of Candida spp. was isolated after $24 \mathrm{~h}$ of incubation from both synovial fluid and the fragments of synovial tissue. The identification of Candida spp. was based on phenotypic feature such as a description of the macro- and micromorphology and through fermentation of carbohydrates and auxanographic using a commercial yeast identification (Api $20 \mathrm{C}$ Aux Biomerieux ${ }^{10}$ ). Colonies grown on Sabouraud dextrose agar were subjected to polymerase chain reaction (PCR) amplification and sequencing analysis in order to determine the Candida species. One fungal colony was transferred in a $1.5 \mathrm{ml}$ tube containing $200 \mu \mathrm{l}$ of distillated water. DNA was extracted using the QIAamp DNA mini kit $^{11}$ in accordance to manufacturer's instructions and the D1/D2 region of the 26S rRNA gene was amplified by PCR assay, as previously described [14]. PCR positive reactions were purified using Wizard SV Gel and PCR Clean-up System (Promega, Madison, WI, U.S.A.) in accordance with the manufacturer's recommended protocol and subjected to direct sequencing. Consensus sequences were created with the BioEdit

\footnotetext{
${ }^{10}$ Api 20 C Aux Biomerieux, Marcy-l'Étoile, France.

11 Qiagen, Hilden, Germany.
}

Sequence Alignment Editor Software v 7.0.9.0 and then searched against the Genbank database. The isolated Candida sp. was thereby identified as C. guilliermondii.

Before treatment was initiated with antifungal drugs, sampling of synovial material was repeated with the same aseptic procedure and growth of C. guilliermondii was confirmed. Culturing of blood and urine samples did not produce Candida colonies and consequently systemic candidiasis was rejected. Antifungal therapy was started with fluconazole ${ }^{12}$ (5 mg/kg per oral [PO] for 10 days; once a day). The intra-articular treatment was performed under general anaesthesia: after premedication with dexmedetomidine (see footnote 1$)(4 \mu \mathrm{g} / \mathrm{kg}$ Intramuscular [IM]) and methadone (see footnote 3) $(0.2 \mathrm{mg} / \mathrm{kg} \mathrm{IM})$, general anaesthesia was induced with propofol (see footnote 5) (3 mg/kg IV), and maintained as previous described. The left hind limb was then prepared for aseptic procedure and copious joint lavage $(30 \mathrm{ml} / \mathrm{kg}$ of lactated Ringer's solution) and intra-articular administration of $10 \mathrm{ml}(0.7 \mathrm{mg} / \mathrm{kg})$ of $0.2 \%$ fluconazole $^{13}$ solution were performed on alternate days for three times. Synovial fluid analysis after 9 days of treatment, failed to culture C. guilliermondii. Two weeks following initiation of intraarticular treatment, clinical examination revealed significant improvement in function with $1 / 5$ left hind limb lameness (lameness barely detectable) and no resentment to manipulation of the left stifle. Radiography of the stifle revealed a similar grade of osteoarthritis but an evident decrease of synovial volume. Systemic treatment for leishmaniasis was instituted. New samples of synovial fluid from the left stifle were collected during TTA 1 month later and sent to the same diagnostic laboratory involved for previous testing. These samples were negative for bacterial and fungal growth (serial clinical procedures and treatments carried out are listed in Table 2). Six month after TTA surgery the referring veterinarian reported that the dog showed a clinical improvement with no lameness $(0 / 5)$.

Candida spp. are opportunistic pathogens often causing severe systemic infections in immunocompromised patients $[15,16]$. In humans, Candida spp. inducedarthritis is a rare disease and most of the cases have been also associated with immunosuppressive conditions such

\footnotetext{
${ }^{12}$ Diflucan ${ }^{\circledR}$, Pfizer, New York, USA.

${ }^{13}$ Fluconazolo ratiopharm $^{\circledR}$, Ratiopharm Italia Srl, Assago, Italia.
} 


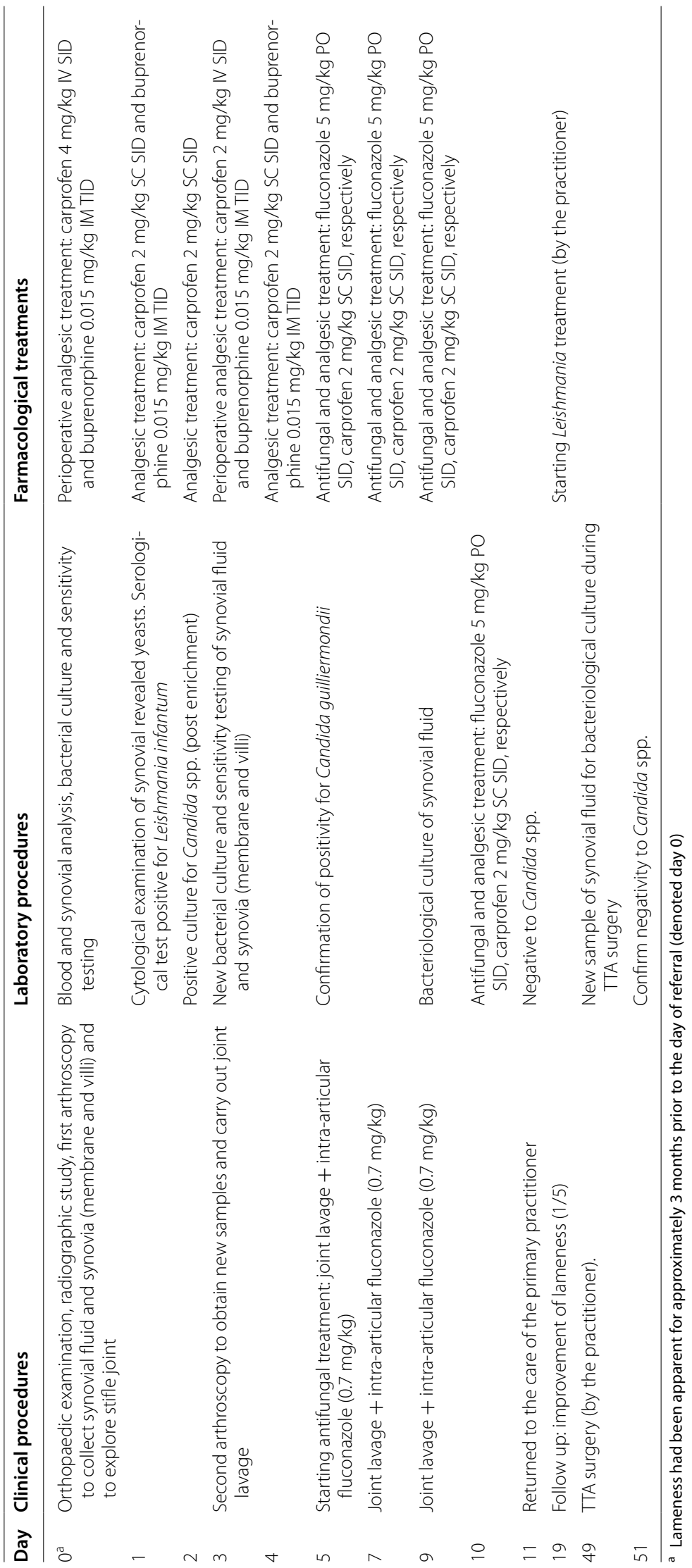


as cancer, AIDS, organ transplantation, chronic renal failure, steroid use, and heroin abuse [17-19]. Although virtually any joint can be affected, large, weight-bearing joints such as the knees are most commonly involved [15]. Candida guillermondii is widely distributed in nature, frequently isolated from the environment and it is a part of saprophyte human and animal microflora on the skin and mucosal surfaces. However, it is considered to be an uncommon causative agent of disease [20]. Reports of Candida spp. infections in dogs are rare as well. Candida guilliermondii was isolated from cutaneous candidiasis in a dog [21]. Systemic candidiasis has been reported in dogs causing signs of acute onset with pyrexia and septic shock [22, 23, 24]. Localized candidiasis is often asymptomatic [17], although in chronically immunosuppressed dogs with involvement of the skin, nail beds, urinary tract, ears, and gastrointestinal tract have been described $[1,25]$. A single report describes an intra-articular fungal (Blastomyces spp.) infection in the carpus of a Labrador retriever, which was treated successfully with itraconazole PO [26]. To the authors' knowledge this is the first report of a C. guilliermondii joint infection in a dog. Candida spp. infections have been associated with immunosuppression in dogs and humans [15]. Four mechanisms may have contributed to the development of C. guilliermondii infection in the present case: leishmaniasis, intra-articular steroids [27], repeated needle puncture compromising the skin barrier [4,7], and lymphoplasmacytic synovitis observed especially in Boxer dogs [28]. Although minimally invasive techniques such as joint puncture are not an important risk factor for developing infective arthritis, in our opinion joint injections pose a potential risk for microbiological joint contamination that may evolve in infection especially in attenuated immune response dogs. Intra-articular injections of glucocorticoids are widely used in arthritic human patients $[29,30]$, despite some evidence that intra-articular steroids do not influence the expression of some important mediators of inflammation and cartilage destruction $[31,32]$. It has been reported that bacterial DNA is frequently found in canine synovial tissue from cranial cruciate ligament rupture joints, possibly as a result of altered immune defences, which may relate to lymphoplasmacytic inflammation [28]. In the current case, leishmaniasis may have contributed to destabilize the dog's immune system [33] by promoting the rooting of the $C$. guilliermondii. Moreover, intra-articular glucocorticoids may also have induced a status of local immune suppression that may have created a favourable environment for C. guillermondii. In the authors' opinion both previously performed intra-articular treatments and the instability due to the cruciate deficiency, maybe have had major roles among the predisposing factors for developing joint fungal infections. In our opinion the elimination of C. gulliermondii was indicated prior to treatment of the leishmaniasis due to the possible side effects and toxicity of drugs resulting from the combined treatment. The therapy of leishmaniasis was appropriate prior to surgery to stabilize the stifle in order to reduce the complications that may arise by the oxidative stress caused by the surgical procedure in an immunocompromised patient. Optimal treatment of C. guilliermondii arthritis has not been established [17]. Antifungal susceptibility testing is usually unstandardized and unreliable [34], so antifungal agents that are effective against a broad spectrum of medically important Candida spp. are recommended. In one recent report, a woman with Candida spp. infection of a joint was treated with joint debridement and antifungal medications, such as amphotericin $B$ $(0.5-1 \mathrm{mg} / \mathrm{kg} /$ day IV) and fluconazole (400 mg/day PO) [17]. Amphotericin was given IV for 3 weeks and PO fluconazole was prescribed for 6 months. Amphotericin B is a polyene antifungal commonly used for overwhelming systemic mycoses and leishmaniosis [1]. Fluconazole is an antifungal drug with higher affinity for fungal enzymes in comparison to ketoconazole or itraconazole. Its bioavailability is elevated after IV or PO administration [1]. A long-term survey of Candida spp. infections demonstrated that fluconazole had fewer side effects and a superior antifungal effect than amphotericin B [35]. Treatment of a Candida spp. joint infection using fluconazole as a sole agent has been reported in a single human [36]. In human medicine, amphotericin B may have poor absorption after oral administration and is painful when injected IM, potentially nephrotoxic and can exacerbate liver failure. For this reason we chosen fluconazole as treatment of the localized fungal infection. Dosage recommendations vary from 2.5 to $10 \mathrm{mg} / \mathrm{kg}$ every $12-24 \mathrm{~h}$ for treatment of candidiasis in humans. In this case fluconazole was dosed at $5 \mathrm{mg} / \mathrm{kg}$ once a day, which resulted in an effective response [22]. Even in neonatal foals fluconazole (4-5 mg/kg PO) has been used to treat systemic candidiasis and a lower toxicity than amphotericin B was shown [5].

A variety of treatments are available for septic arthritis, but long-term osteoarthritis is inevitable. The treatment options range from systemic drugs, local and regional medications, joint lavage via large bore needles, and arthroscopic lavage, to open arthrotomies for continuous drainage $[7,37]$. Although joint lavage has not been found to be effective in slowing the rate of progression of osteoarthritis without sepsis, it is recommended in septic arthritis to remove some of the inflammatory mediators and debris [37]. Large portals allow the removal of purulent debris and the use of a large volume of fluid. Repeated lavage or continuous drainage of the joint may 
be indicated if clinical signs persist from continued synovitis or production of purulent debris [37]. The decision for choosing the intra-articular route for fluconazole administration was based on the aim to obtain a high concentration of compound in the joint and eliminate the infection more rapidly. This may be debated since in humans a parenteral administration of fluconazole produces substantial synovial fluid levels [38]. In this case we did not observe joint swelling or localized pain nor any other side effects in the following days after administration. As reported in horses [4] intra-articular administration of fluconazole can be actuated without local/ systemic complications. Candida spp. joint infections need treatment in all cases. Since Candida spp. are less potent infectious agents in a healthy immunocompetent patient, the presence of Candida spp. infection often indicates a status of immune deficiency. A combined approach for treatment of the Candida infection and of the pathology responsible for immunocompromised is necessary.

\section{Conclusions}

To the authors' knowledge, this is the first documented case of C. guilliermondii joint infection in a dog. The objective of this study was to document the successful diagnosis and treatment of fungal infectious arthritis. Since this is the first report on Candida spp. joint infection in a dog, no guidelines regarding treatment exist and the decision to administer fluconazole into the joint was made mainly on reports in horses. Fungal arthritis should be considered when encountered by joint infections, particularly in cases with a concomitant immunosuppression. In fact, the diagnosis of septic arthritis with a normally commensal fungal organism should alert the clinician to the possibility of local and/or systemic immunocompromised state. Treatment including repeated joint lavage and administration of systemic and local (intra-articular) fluconazole resulted in eradication of the C. guilliermondii infection in this case.

\section{Abbreviations}

IV: intravenous; IM: intramuscular; PO: per os/per oral; SC: subcutaneous; TTA: tibial tuberosity advancement.

\section{Authors' contributions}

$A B$ carried out the treatment and critically revised the manuscript for important intellectual content. CM carried out the treatment and drafted the manuscript; GM and AC participated in drafting the manuscript; VS carried out the laboratory work; RKS agrees to be accountable for all aspects of the work ensuring that questions related to the accuracy or integrity of any part of the work are appropriately investigated and resolved; FP wrote the laboratory findings in the manuscript; All authors read and approved the final manuscript.

\section{Author details}

${ }_{1}^{1}$ Department of Veterinary Medicine, University of Perugia, Via S. Costanzo n.4, 06126 Perugia, PG, Italy. ${ }^{2}$ Clinica Veterinaria Orvieto, Strada del Piano 9,
05018 Orvieto Scalo, TR, Italy. ${ }^{3}$ Institute of Infectious Diseases and Zoonoses, Faculty of Veterinary Medicine, Ludwig Maximilians Universität Munich, Munich, Germany.

\section{Acknowledgements}

The authors would like to thank Dr. Silvia Crotti, Istituto Zooprofilattico Sperimentale Umbria e Marche, Perugia (Italy) for her assistance in the molecular biology assay.

\section{Competing interests}

The authors declare that they have no competing interests.

Received: 9 March 2016 Accepted: 30 June 2016

Published online: 08 July 2016

\section{References}

1. Greene CE, Chandler FW. Candidiasis, torulopsosis, and rhodotorulosis. In: Greene CE, editor. Infectious diseases of the dog and cat. 3rd ed. Philadelphia: Saunders Elsevier; 2006. p. 627-33.

2. Habib GS, Saliba W, Nashashibi M. Local effects of intra-articular corticosteroids. Clin Reumathol. 2010;29:347-56.

3. Silveira LH, Cuellar ML, Citera G, Cabrera GE, Scopelitis E, Espinoza LR. Candida arthritis. Rheum Dis Clin North Am. 1993;19:427-37.

4. Cohen JM, Ross MV, Busschers E. Diagnosis and management of Candida utilis infectious arthritis in a Standardbred filly. Equine Vet Educ. 2008;20:348-52.

5. Reilly LK, Palmar JE. Systemic candidiasis in four foals. J Am Vet Med Ass. 1994;205:464-6.

6. Peremans K, Verschooten F, Moor A, Desmet P. Monoarticular infectous arthritis in the horse: 35 cases. J Equine Vet Sci. 1991;11:27-32.

7. Lapointe JM, Laverty S, Lovoie JP. Septic arthritis in 15 Standardbred racehorses after intra-articular injection. Equine Vet J. 1992;24:430-4.

8. Schneider RK, Bramlage LR, Mecklenburg LM, Moore RM, Gabel AA. A retrospective study of 192 horses affected with septic arthritis/tenosynovitis in horses. Equine Vet J. 1992;24:436-42.

9. Quinn MM, Keuler NS, Lu Y, Faria MLE, Muir P, Markel MD. Evaluation of agreement between numerical rating scales, visual analogue scoring scales, and force plate gait analysis in dogs. Vet Surg. 2007;36:360-7.

10. Carobbi B, Ness MG. Preliminary study evaluating tests used to diagnose canine cranial cruciate ligament failure. J Small Anim Pract. 2009;50:224-6.

11. Bufalari A, Maggio C, Cerasoli I, Morath U, Adami C. Preemptive carprofen for peri-operative analgesia in dogs undergoing Tibial Plateau Leveling Osteotomy (TPLO): a prospective, randomized, blinded, placebo controlled clinical trial. Schweiz Arch Tierh. 2012;154:105-11.

12. Pozzi A, Hildreth BE, Rajala-Schultz PJ. Comparison of arthroscopy and arthrotomy for diagnosis of medial meniscal pathology: an ex vivo study. Vet Surg. 2008;37:749-55.

13. Beale BS, Hulse DA, Schulz KS, Whitney WO. Small animal arthroscopy. Philadelphia: Saunders; 2003. p. 50-79.

14. Yang $Q$, Zhang $H$, Li X, Wang Z, Xu Y, Ren S, et al. Extracellular enzyme production and phylogenetic distribution of yeasts in wastewater treatment systems. Bioresour Technol. 2013;129:264-73.

15. Kholi R, Hadley S. Fungal arthritis and osteomyelitis. Infect Dis Clin North Am. 2005;19:831-5.

16. Dimitrova P, Ivanovska N. Host resistance to Candida albicans infection of mice with collagen-induced arthritis treated with leflunomide. Res Microbiol. 2006;157:525-30

17. Lee $\mathrm{CH}$, Oh JM, Oh SR, Yoo M, Lee MS. Candida arthritis after arthroscopic arthroplasty in a patient without predisposing factors. Open Rheumatol J. 2010;4:7-9.

18. Lim KB, Kwak YG, Kim YS, Park KR. Shoulder joint infectious arthritis and acromioclavicular joint osteomyelitis due to Candida. Ann Rehabil Med. 2012;36:573-7.

19. Jeong YM, Cho HY, Lee SW, Hwang YM, Kim YK. Candida septic arthritis with rice body formation: a case report and review of literature. Kor J Radiol. 2013;14:465-9.

20. Savini V, Catavitello C, Onofrillo D, Masciarelli G, Astolfi D, Balbinot A, et al. What do we know about Candida guilliermondii? A voyage throughout 
past and current literature about this emerging yeast. Mycoses. 2010;54:434-41.

21. Mueller RS, Bettenay SV, Shipstone M. Cutaneous candidiasis in a dog caused by Candida guilliermondii. Vet Rec. 2002;23:728-30.

22. Ong RK, Raisis AL, Swindells KL. Candida albicans peritonitis in a dog. J Vet Emerg Crit Care. 2010;20:143-7.

23. Rogers CL, Gibson C, Mitchell SL, Keating JH, Rozanski EA. Disseminated candidiasis secondary to fungal and bacterial peritonitis in a young dog. J Vet Emerg Crit Care. 2009;19:193-8.

24. Heseltine JC, Panciera DL, Saunders GK. Systemic candidiasis in a dog. J Am Vet Med Assoc. 2003:223:821-4

25. Pressler BM, Vaden SL, Lane IF. Candida spp. urinary tract infections in 13 dogs and seven cats: predisposing factors, treatment, and outcome. J Am Anim Hosp Assoc. 2003;39:263-70.

26. Woods KS, Barry M, Richardson D. Carpal intra-articular blastomycosis in a Labrador retriever. Can Vet J. 2013;54:167-70.

27. Charalambous M, Tryfonidis M, Sadiq S, Hirst P, Paul A. Septic arthritis following intra-articular steroid injection of the knee-a survey of current practice regarding antiseptic technique used during intra-articular steroid injection of the knee. Clin Rheumatol. 2003;22:386-90.

28. Erne JB, Goring RL, Kennedy FA, Schoenborn WC. Prevalence of lymphoplasmacytic synovitis in dogs with naturally occurring cranial cruciate ligament rupture. J Am Vet. Med Assoc. 2009;235:386-90.

29. Klint E, Grundtman C, Engstro M, Catrina Al, Makrygiannakis D, Klareskog $L$, et al. Rutin has therapeutic effect on septic arthritis caused by Candida albicans. Int Immunopharmacol. 2009;9:207-11.

30. Cunnington J, Marshall N, Hide G, Bracewell C, Isaacs J, Platt P, et al. A randomized, double-blind, controlled study of ultrasound-guided corticosteroid injection into the joint of patients with inflammatory arthritis. Arthritis Rheum. 2010;62:1862-9.
31. Ulfgren AK. Intraarticular glucocorticoid treatment reduces inflammation in synovial cell infiltrations more efficiently than in synovial blood vessels. Arthritis Rheum. 2005;52:3880-9.

32. Young L, Katrib A, Cuello C, Vollmer-Conna U, Bertouch JV, RobertsThomson PJ, et al. Effects of intraarticular glucocorticoids on macrophage infiltration and mediators of joint damage in osteoarthritis synovial membranes findings in a double-blind, placebo-controlled study. Arthritis Rheum. 2001;44:343-50.

33. Reis AB, Giunchetti RC, Carrillo E, Martins-Filho OA, Moreno J. Immunity to Leishmania and the rational search for vaccines against canine leishmaniasis. Trends Parasitol. 2006;26:341-9.

34. Anaissie E, Paetznick V, Bodey GP. Fluconazole susceptibility testing of Candida albicans: microtiter method that is independent of inoculum size, temperature, and time of reading. Antimicrob Agents Chemother. 1991:35:1641-6.

35. Horn DL, Neofytos D, Anaissie EJ, Fishman JA, Steinbach WJ, Olyaei AJ, et al. Epidemiology and outcomes of candidemia in 2019 patients: data from the prospective antifungal therapy alliance registry. Clin Infect Dis. 2009;48:1695-703.

36. Merrer J, Dupont B, Nieszkowska A, De Jonghe B, Outin H. Candida albicans prosthetic arthritis treated with fluconazole alone. J Infect. 2001;42:208-9.

37. Hewes CA, Macintire DK. Intra-articular therapy to treat septic arthritis in a dog. J Am Anim Hosp Assoc. 2011;47:280-4.

38. Rex JH, Walsh TJ, Sobel JD, Filler SG, Pappas PG, Dismukes WE, et al. Practice guidelines for the treatment of candidiasis. Clin Infect Dis. 2000;30:662-78.

\section{Submit your next manuscript to BioMed Central and we will help you at every step:}

- We accept pre-submission inquiries

- Our selector tool helps you to find the most relevant journal

- We provide round the clock customer support

- Convenient online submission

- Thorough peer review

- Inclusion in PubMed and all major indexing services

- Maximum visibility for your research

Submit your manuscript at www.biomedcentral.com/submit
Biomed Central 